\title{
Decisions, Decisions: Noise and its Effects on Integral Monte Carlo Algorithms
}

\author{
J. D. Doll \\ Department of Chemistry, Brown University, Providence, RI 02912 \\ D. L. Freeman \\ Department of Chemistry, University of Rhode Island, Kingston, RI 02881
}

\begin{abstract}
In the present paper we examine the effects of noise on Monte Carlo algorithms, a problem raised previously by Kennedy and Kuti (Phys. Rev. Lett. 54, $2473(1985))$. We show that the effects of introducing unbiased noise into the acceptance/rejection phase of the conventional Metropolis approach are surprisingly modest, and, to a significant degree, largely controllable. We present model condensed phase numerical applications to support these conclusions.
\end{abstract}

Typeset using REVTEX 


\section{INTRODUCTION}

As emphasized by Ulam in his autobiography [1], the problem of decision making in a statistical environment is an interesting issue. When procedures or input are corrupted by "noise," the resulting outcomes will in general differ from their zero noise counterparts. Such modifications may range from inconsequential to extreme depending on the details of the algorithm and noise involved.

Questions related to the spirit of this general issue arise when we consider the effects of noise on integral Monte Carlo techniques [2]. In these methods "decisions" are made at various stages according to a relatively simple set of rules. Our concern in the present paper is how the inclusion of various types of noise into these decision making steps alters the

predictions of such algorithms. In view of its practical importance, we focus attention in the present discussion on the venerable "Metropolis" procedure [3].

Monte Carlo algorithms in general are among the most widely utilized numerical procedures in science [4]. They have become, for example, a primary tool for the analysis of many-body phenomena. The techniques are broadly applicable to both classical [5] and quantum-mechanical [6] problems.

Beyond their immediate numerical utility, these statistical methods have served to promote the development of new approaches to a diverse range of important problems. In the general field of optimization, for instance, the simulated annealing method [7] has emerged as a valuable technique for function minimization. This approach attempts to find the minimum of a specified function by recasting the problem as one of finding the zero "temperature" structure of a fictitious classical system. The "potential energy" of this system is taken to be the function whose minimum is being sought. As emphasized by Press, et al. [8], the resulting method is a relatively robust one in that the thermal fluctuations involved assist in avoiding local minima. Related "quantum annealing" approaches have also been reported recently [9-11].

The typical use of of the integral Monte Carlo methods considered here is to obtain 
statistical estimates of generalized averages that arise in various physical applications. Integral Monte Carlo methods, in general, approximate such averages with statistical estimates based on a finite set of quadrature points drawn randomly from the probability distribution functions in question. The Metropolis method [3], in particular, generates these points by devising a random walk that visits each configuration space location with a probability that is proportional to its associated probability. If "unbiased," these estimates can be made arbitrarily exact by increasing the scale of the random walk. Methods suitable for treating "rare event" situations [12 and for coping with "sparse" distributions [13,14 are described elsewhere.

In typical integral Monte Carlo applications, the distribution function involved is known with certainty. In other applications, however, the information necessary to implement the Monte Carlo procedure is available only numerically and only with limited precision [15]. It is thus important to clarify the effects of such "noise" on the basic algorithms involved if we are to utilize conventional methods in these instances.

The outline of the remainder of present paper is as follows: Starting with a brief review of the Metropolis method, we present in Section II a number of results concerning the influence of noise on this procedure. In Section III we document the principal conclusions of Section II with prototypical numerical applications related to the study of the dynamics of condensed-phase quantum-mechanical systems.

\section{FORMAL DEVELOPMENTS}

Detailed balance is at the core of the Metropolis algorithm. In particular, the requirement that

$$
\rho(A) P(A \rightarrow B)=\rho(B) P(B \rightarrow A)
$$

is a sufficient condition to assure that a random walk based on the transitional rules, $P(X \rightarrow$

$Y)$, visits points, $\mathrm{Y}$, in proportion to their associated equilibrium densities, $\rho(Y)$. Rewritten 
slightly, the detailed balance constraint requires that the transition rules underlying the Monte Carlo random walk satisfy

$$
\frac{P(A \rightarrow B)}{P(B \rightarrow A)}=\frac{\rho(B)}{\rho(A)}
$$

There are many possible choices for transition probabilities consistent with Eq. (2). The original Metropolis method [3] takes

$$
P(A \rightarrow B)=M\left(\rho_{B} / \rho_{A}\right)
$$

where the $M(x)$ is the "Metropolis" function,

$$
M(x)=\min (1, x) .
$$

For later purposes, it is convenient to take $M(x)$ to be zero if $x$ is negative. It is easy to verify that

$$
M(x) / M(1 / x)=x
$$

in accordance with the detailed balance.

For typical problems, well established procedures exist to generate the random walks specified by Eq. (3). The elemental building block for such walks is the combination of randomly generated "trial" moves followed by "acceptance/rejection" decisions. Descriptions of these procedures are given elsewhere [2,3]. The essential points for the present discussion are: (1) the decisions involved are the mechanism by which detailed balance is enforced, and (2) the decisions are based on equilibrium density ratios.

When we no longer know the required density ratios precisely but rather possess only noisy, statistical estimates of the required information, it is not immediately obvious how best to proceed. One obvious approach is to ignore the presence of noise and simply implement the Metropolis procedure in its original form. If we do so, however, we have no assurance the resulting method will satisfy detailed balance. In particular, the transition probability for such an approach, $P_{0}(A \rightarrow B)$, can be shown to be, 


$$
P_{0}(A \rightarrow B)=\int d r M(r) f(r)
$$

where $f(r)$ denotes the probability that the B/A density ratio for the step assumes the value $r$. Equation (6) obviously reduces to Eq. (3) for probability distributions sharply peaked about the true ratio, $\rho(B) / \rho(A)$, In general, however, $P_{0}$ differs from its zero noise counterpart and the guarantee of detailed balance is lost. The influence of noise on the Metropolis method can thus be explored by examining its effects on the transition probability (Eq. (6)) and on the associated detailed balance ratios (Eq. (2)). Because of its importance in practical applications, we assume in what follows that $f(r)$ in Eq. (6) is a normalized gaussian with a mean equal to the exact density ratio.

To explore the questions raised above, we show in Figs. (1a) and (1b) plots of the transition probabilities produced by Eq. (6). These were obtained assuming gaussian noise of fixed absolute (Fig. (1a)) and relative widths (Fig. (1b)) of a particular magnitude (0.2) in the density ratio. For a given absolute noise, we see in Fig. (1a) that $P$ and $P_{0}$ are qualitatively similar, differing only in the regions where the density ratio is near zero or unity. The widths of the regions where differences are observed are approximately the width of $f(r)$. It is easy to see from the convolution structure of Eq. (6) that this is a general result. Explicitly, the average involved in Eq. (6) modifies the resulting transition probability relative to the zero noise limit only in regions where the Metropolis function is neither constant nor purely linear over the width of the gaussian noise. For this reason, the effects of a fixed relative error in the acceptance/rejection density ratio (Fig. (1b)) are confined to the density ratio region near unity.

While it is informative to examine the behavior of the individual transition probabilities themselves (Fig. (1)), it is important to emphasize that the primary measure of the effect of noise on the Metropolis method is its consequence for detailed balance. In Figs. (2a) and (2b) we display plots of $P_{0}(x) / P_{0}(1 / x)$ for a range of density ratios, $x$, for the systems examined in Fig. (1). Breakdowns in detailed balance are signaled in such a plot by deviations from linearity. In contrast to the behavior of the individual transition probabilities in Fig. (1a), 
we see in Fig. (2a) that effects of absolute noise in the density region near unity tend to cancel from the detailed balance condition. Evaluating Eq. (6) analytically for noise of a given width, $\sigma$, it is straightforward to show that for small widths the effects of noise are minimal over the density ratio range $\sigma \leq x \leq 1 / \sigma$. From Fig. (2b) we see that relative noise in the acceptance/rejection ratio has little effect on the detailed balance ratio in any region. As will be documented below, these conclusions remain valid for a surprisingly large range of absolute and relative noise.

The simple analysis presented above suggests that the Metropolis method is robust with respect to the inclusion of unbiased noise to its decision making steps. Even rather sizeable relative errors in the acceptance/rejection ratio appear to have little effect on detailed balance and hence on the overall performance of the method. While absolute errors have somewhat more pronounced consequences, their effects are restricted to an identifiable class of trial moves. Specifically, we have seen above that fixed noise enters the Metropolis method principally thorough decisions involving attempted moves between points of grossly dissimilar equilibrium probability. Such moves can be largely avoided in practice by utilizing a trial displacement length scale that is small relative to that characterizing the interaction potential. In the zero noise limit, the selection of such length scales is purely a question of efficiency and any sensible choice will suffice. We see, however, that with the addition of a specified absolute noise that smaller length scales are preferable. Their use will assure that the preponderance of the acceptance/rejection decisions will be made in situations where the effects of noise are essentially inconsequential.

\section{NUMERICAL EXAMPLE}

We present below a brief numerical example chosen to illustrate the potential use of the ideas discussed in the previous section in study of quantum dynamics. We examine a model system [16] that consists of a single moving particle trapped on a line between

two space-fixed particles (separated by a distance L) that act as a Rcage.S The particle 
whose dynamics is of interest is assumed to interact with the fixed atoms through pairwise Lennard-Jones potentials. These potentials as well as the cage dimensions can be varied to probe the behavior of various prototypical systems.

We focus in the following on a quantity that plays a central role in describing the spectroscopy and transport in finite temperature dynamical systems, the "self correlation" function [17]. For single particle systems, this quantity can be defined as

$$
G(y, t)=\left\langle\delta\left(y-\left(x^{\prime}-x\right)\right)\right\rangle
$$

where the brackets in Eq. (7) denote an average over the probability distribution $\rho(x) P\left(x^{\prime} t / x\right)$. Here $\rho(x)$ describes the equilibrium density as a function of moving atom position while $P\left(x^{\prime} t / x\right)$ denotes to the conditional probability that a system initially at position $x$ at time zero is at position $x^{\prime}$ a time $t$ later. Generalizations to many-body situations are straightforward. The self correlation function probes the likelihood of dynamical displacements of a specified size in a finite temperature system. Quantum-mechanical expressions for the required equilibrium density and dynamical transition probability are available [18] in terms of various complex temperature density matrix elements.

Our approach in what follows will be to evaluate Eq. (7) for representative systems via Monte Carlo sampling of the initial and final positions. For molecular systems, the required equilibrium tasks can be performed exactly in general. We thus sample initial positions in what follows in the zero noise limit. The information required to perform $t$ he dynamical sampling task, however, is typically not available directly, but must itself be obtained through a separate calculation. To simulate its possible presence in such calculations, we add noise to the final position sampling process as discussed below.

Figure (3) displays $G(y, t)$ results for the present model where potential and cage parameters were selected to mimic the neon system $\left(\epsilon / k_{B}=35.6 K, \sigma=2.749 \stackrel{\circ}{A}, L=2\left(2^{1 / 6} \sigma\right)\right)$. The necessary quantum-mechanical matrix elements were obtained using NMM methods [19]. The Trotter index in the NMM calculations was taken to be 128, more than adequate to assure numerical convergence. The particular results shown in Fig. (3) correspond to a 
temperature of $51 \mathrm{~K}$ and correspond to the zero noise limit.

Certain general features of $G(y, t)$ are evident in Fig. (3). Classically, the self correlation function approaches a delta function limit as $t \rightarrow 0$. We see in Fig. (3), however, that quantum- mechanical effects produce a broadening of this zero time classical behavior. As time increases near $t=0$, particles move from their starting locations increasing in the width of the corresponding displacement distribution. Ultimately, the "caging effects" of the fixed atoms in the model limit the range of motion and produce the oscillatory structure visible in Fig. (3). Were the caging potential harmonic, the structure would contain a single frequency both classically and quantum- mechanically. Since the potential involved is appreciably anharmonic, however, the temporal structure obtained in Fig. (3) contains several frequency components. Quantum-mechanically, these arise from the interplay between the system's vibrational energy levels while classically they reflect the energy dependence of the vibrational period.

Figures (4) and (5) illustrate the effects of including unbiased gaussian noise of a specified relative and absolute magnitude, respectively, to the sampling of the dynamical transition probability for the system studied in Fig. (3). As expected, we see in Fig. (4) that the effects of including relative noise are minimal for situations where the signal to noise ratio is greater than 2:1. Also as expected, we see in Fig. (5) that the effects of including absolute noise are somewhat more pronounced. When utilized in the Metropolis procedure with step sizes chosen by the usual criterion (50\% acceptance), such noise produces noticeable systematic errors. These effects are largely eliminated, however, when the displacement length scale for the trial moves is reduced as discussed in Section II.

\section{SUMMARY}

We have considered the effects of adding noise to the decision making steps of the Metropolis procedure. Our investigation suggests that this procedure is a relatively robust one with respect to the addition of unbiased noise in the acceptance/ rejection probability 
ratio. Specifically, noise of a specified relative size has little effect on detailed balance and hence on the functioning of the method. While the consequences of a fixed absolute noise

are potentially more severe, we find that such effects are restricted to a particular class of trial moves and are hence relatively easy to minimize in practice.

\section{ACKNOWLEDGMENTS}

The authors would acknowledge support through the National Science Foundation grant NSF- CHE-9203498. DLF also wishes to acknowledge the donors of the Petroleum Research Fund of the American Chemical Society for partial support of this work. 


\section{REFERENCES}

[1] S. M. Ulam, Adventures of a Mathematician (Scribners, New York, 1983), Ch. 15.

[2] See, for example, M. Kalos and P. Whitlock, Monte Carlo Methods (Wiley-Interscience, New York, 1986).

[3] N. Metropolis, A. W. Rosenbluth, M. N. Rosenbluth, A. H. Teller, and E. Teller, J. Chem. Phys. 21, 1087 (1953).

[4] To put this in comment in perspective, computer searches of the scientific literature for the past five years revealed the following number of appearances for some common chemical physics topics and phrases: Monte Carlo (3324), Fourier Transform (2040), ionic (3529), and superconductivity (8807).

[5] See, for example, J. P. Valleau and S. G. Whittington, "A Guide to Monte Carlo for Statistical Mechanics", in Statistical Mechanics, Part A: Equilibrium Techniques, Modern Theoretical Chemistry Series, Vol. 5, B. Berne, Ed. (Plenum, New York, 1976).

[6] For a cross-section of activity, see J. Stat. Phys. 43, 729-1244 (1986).

[7] K. S. Kirkpatrick, C. D. Gelatt and M. P. Vecchi, Science 220, 671 (1983).

[8] W. H. Press, S. A. Teukolsky, W. T. Vetterling and B. P. Flannery, Numerical Recipes, 2nd ed. (Cambridge University Press, Cambridge, 1992).

[9] P. Amara, D. Hsu and J. E. Straub, J. Phys. Chem. 97, 6715 (1993).

[10] J. D. Doll and D. L. Freeman, Computational Science and Engineering, IEEE Computer Society, 1, 22 (1994).

[11] A. B. Finnila, M. A. Gomez, C. Sebenik, C. Stenson, J. D. Doll, Chem. Phys. Letts. 219, 343 (1994).

[12] See, for example, Ref. 2, Ch. 4. 
[13] D. D. Frantz, D. L. Freeman and J. D. Doll, J. Chem. Phys. 93, 2769 (1990).

[14] D. D. Frantz, D. L. Freeman and J. D. Doll, J. Chem. Phys. 97, 5713 (1992).

[15] A. D. Kennedy and J. Kuti, Phys. Rev. Lett. 54, 2473 (1985).

[16] D. L. Freeman, R. D. Coalson and J. D. Doll, J. Stat. Phys., 43, 931 (1986).

[17] D. McQuarrie, Statistical Mechanics (Harper and Row, New York, 1976), pp. 544-553.

[18] J. D. Doll, D. L. Freeman and T. L. Beck, Adv. Chem. Phys. 78, 61 (1990).

[19] D. Thirumalai, E. J. Bruskin and B. Berne, J. Chem. Phys. 79, 5063 (1983). 


\section{FIGURE CAPTIONS}

1. Figure (1a) compares the usual Metropolis function (line with discontinuous slope) with the transition probability obtained from Eq. (6) as a function of the trial probability ratio. Results shown were obtained using gaussian noise with a fixed standard deviation of 0.2 . Figure (1b) is the same as (1a), except that the standard deviation for the noise involved was of a specified relative fraction (20\%) of the probability ratio.

2. Forward and reverse detailed balance quotients for the systems described in Fig. (1) as a function of the trial probability ratio. Breakdowns in linearity signal deviations from detailed balance.

3. The self correlation function (Eq. (7)) for the Lennard-Jones cage problem discussed in Section III. System parameters were those appropriate for neon.

4. The self correlation function for the neon cage problem in Fig. (3) at a specified time (80,000 a.u.). Results shown were obtained from Eq. (7) with various amounts of relative noise $(0 \%-80 \%)$ added to the trial probability ratio.

5. The self correlation function for the neon cage problem in Fig. (3) at a specified time (80,000 a.u.). Zero noise results (exact) are compared with two calculations involving a fixed level of noise (0.4). In the "noisy" calculations, trial step sizes in the final position sampling in Eq. (7) were adjusted to produce $50 \%$ and $80 \%$ acceptance, respectively. 


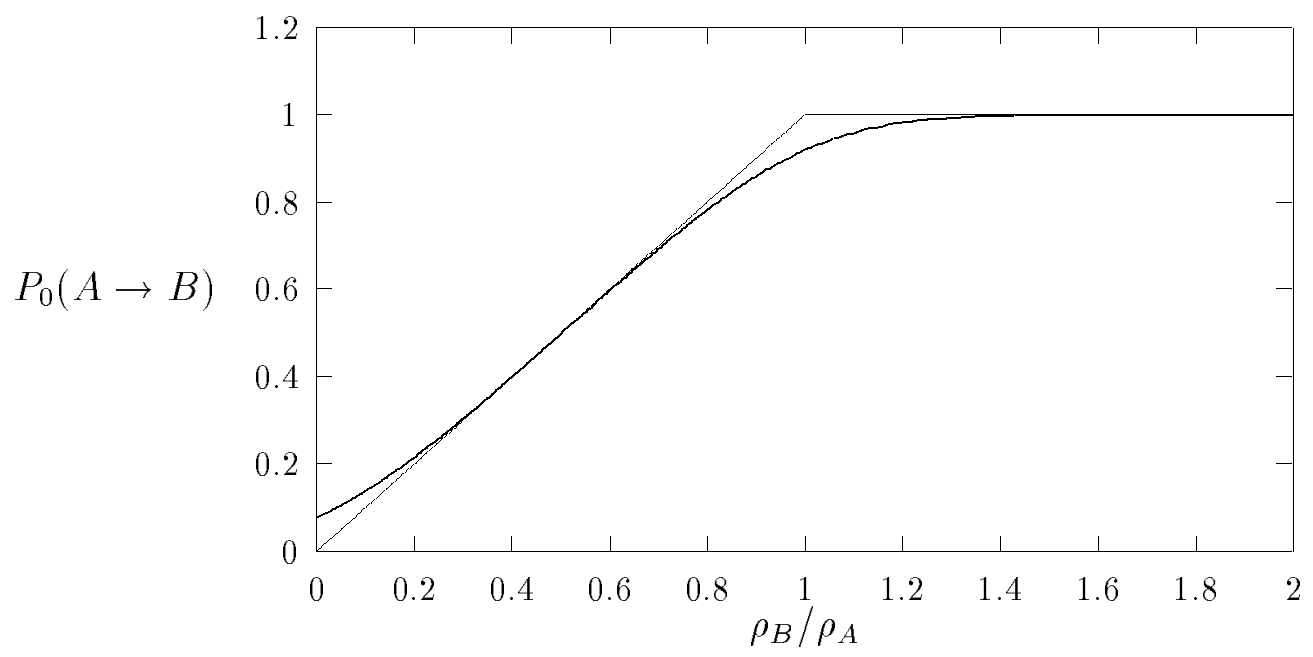

(a)

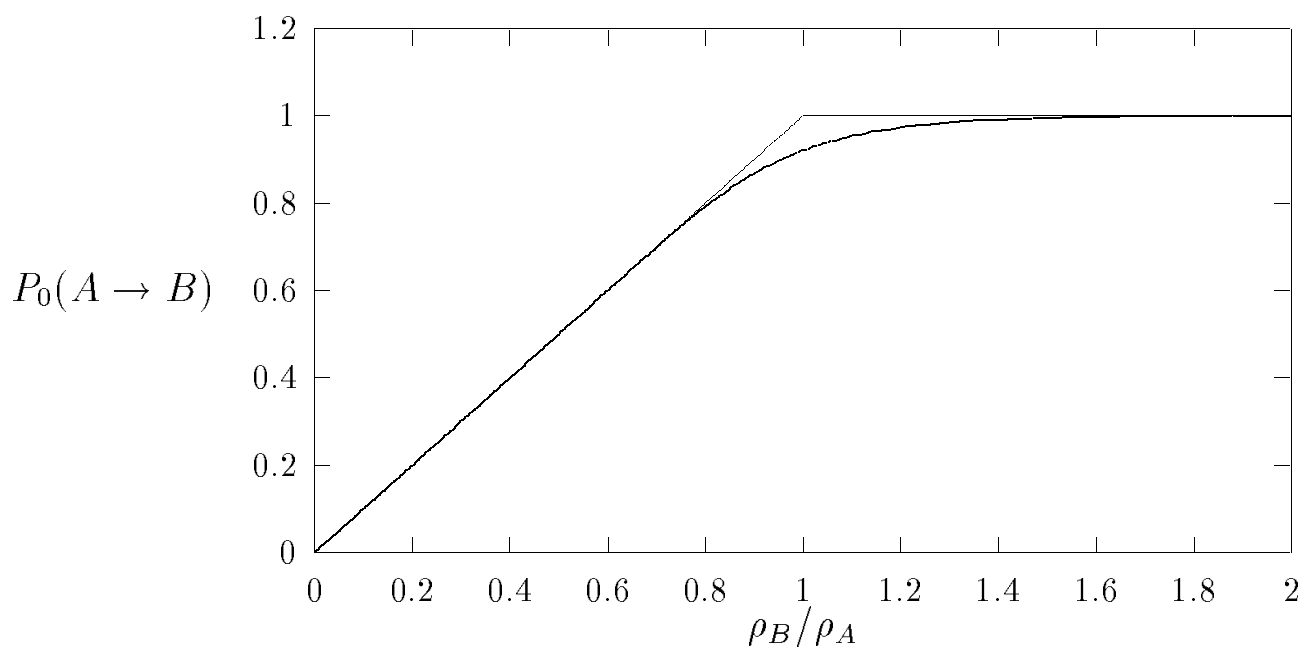

(b) 


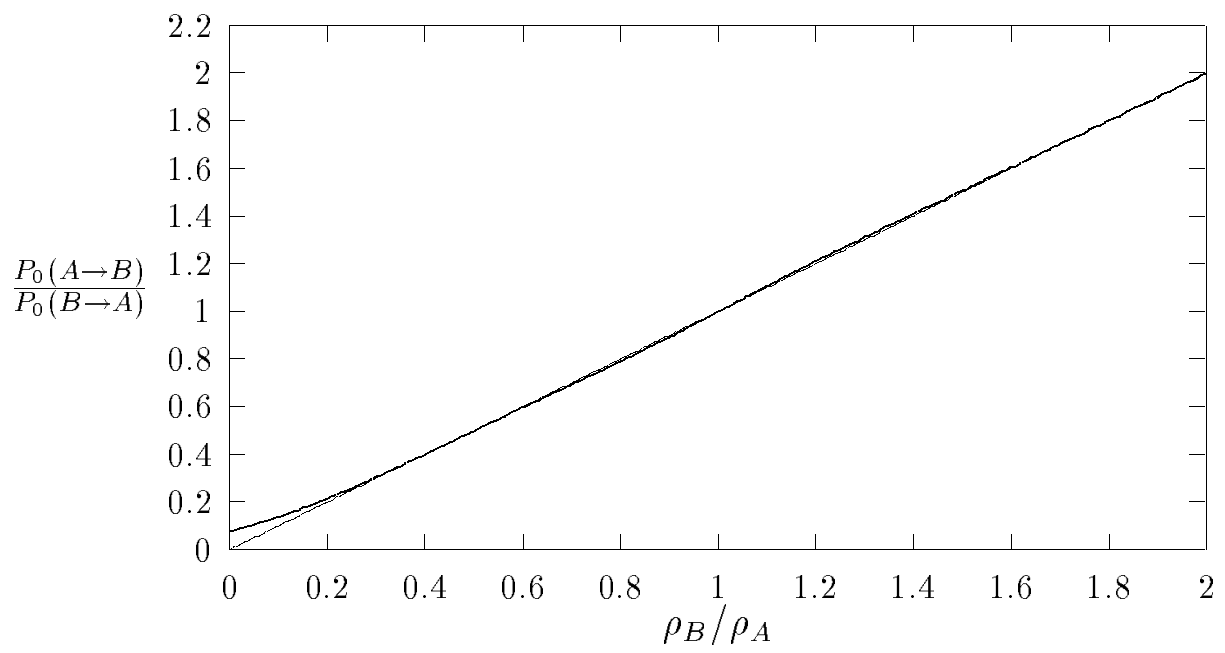

(a)

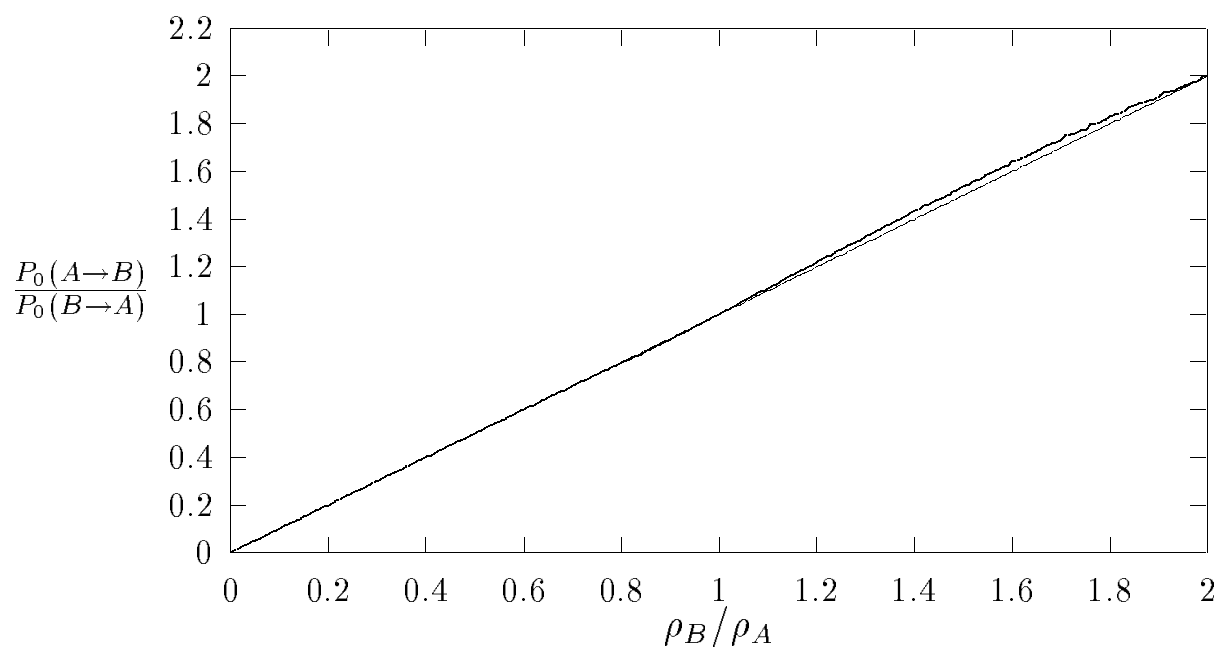

(b) 


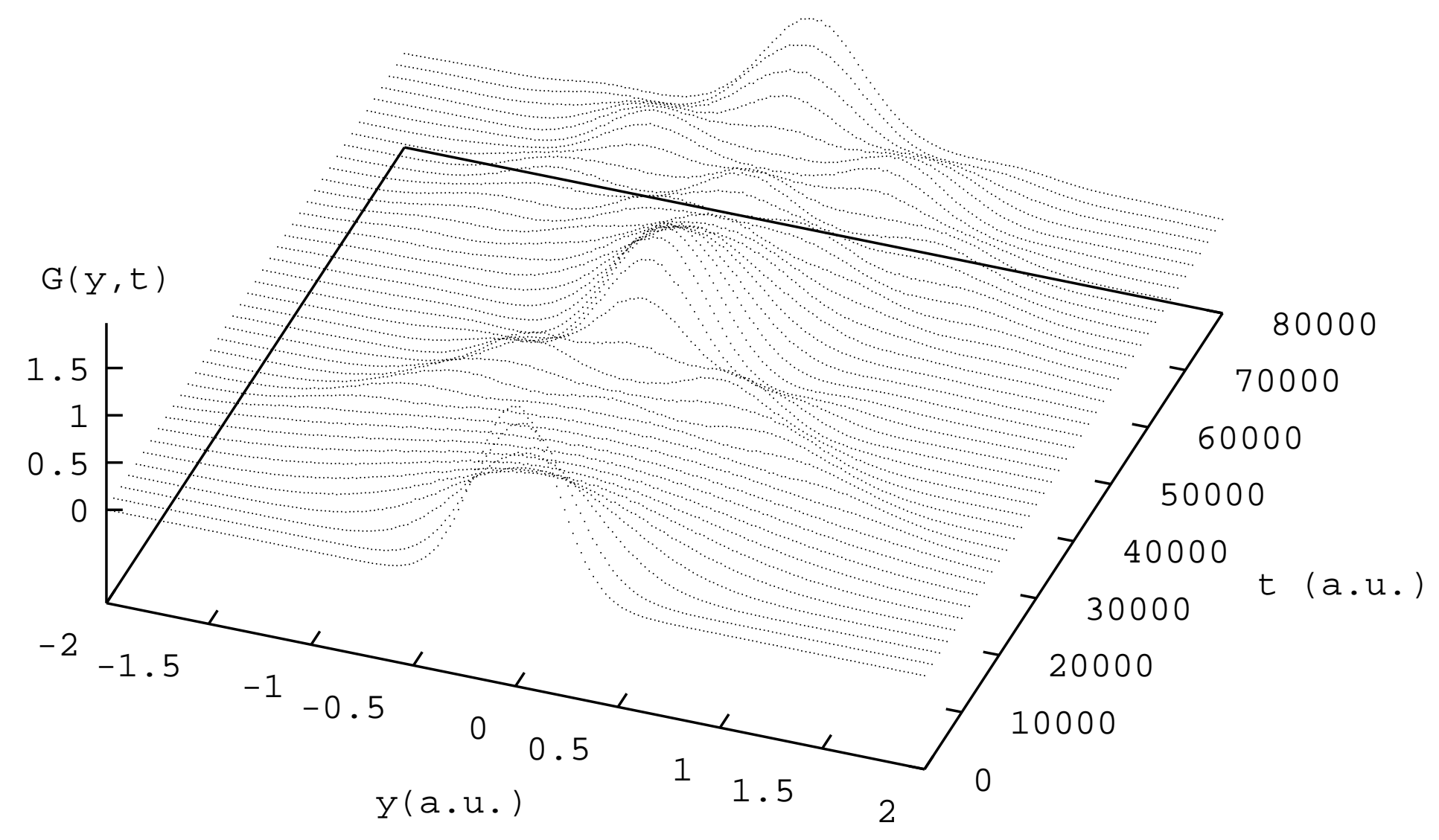




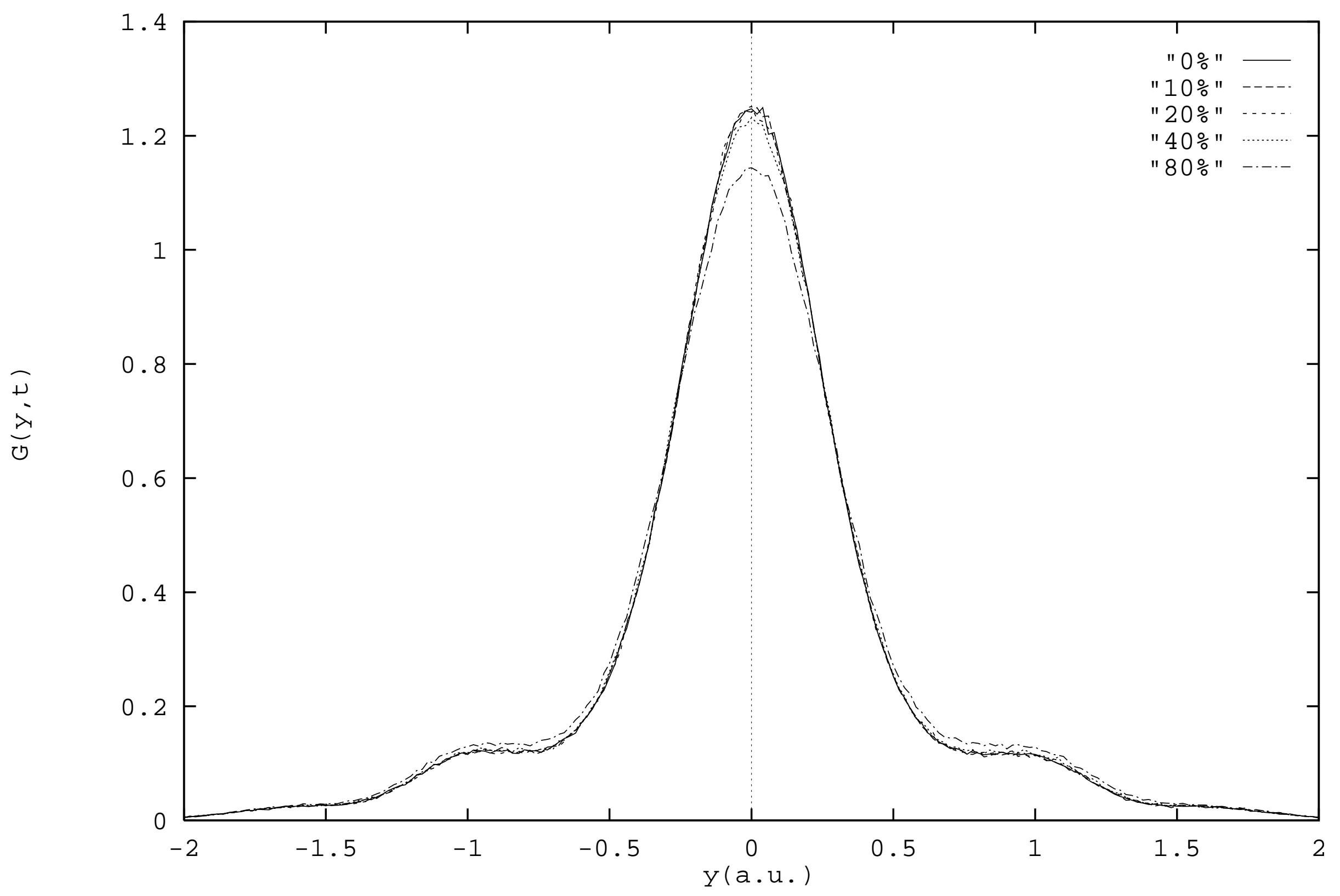




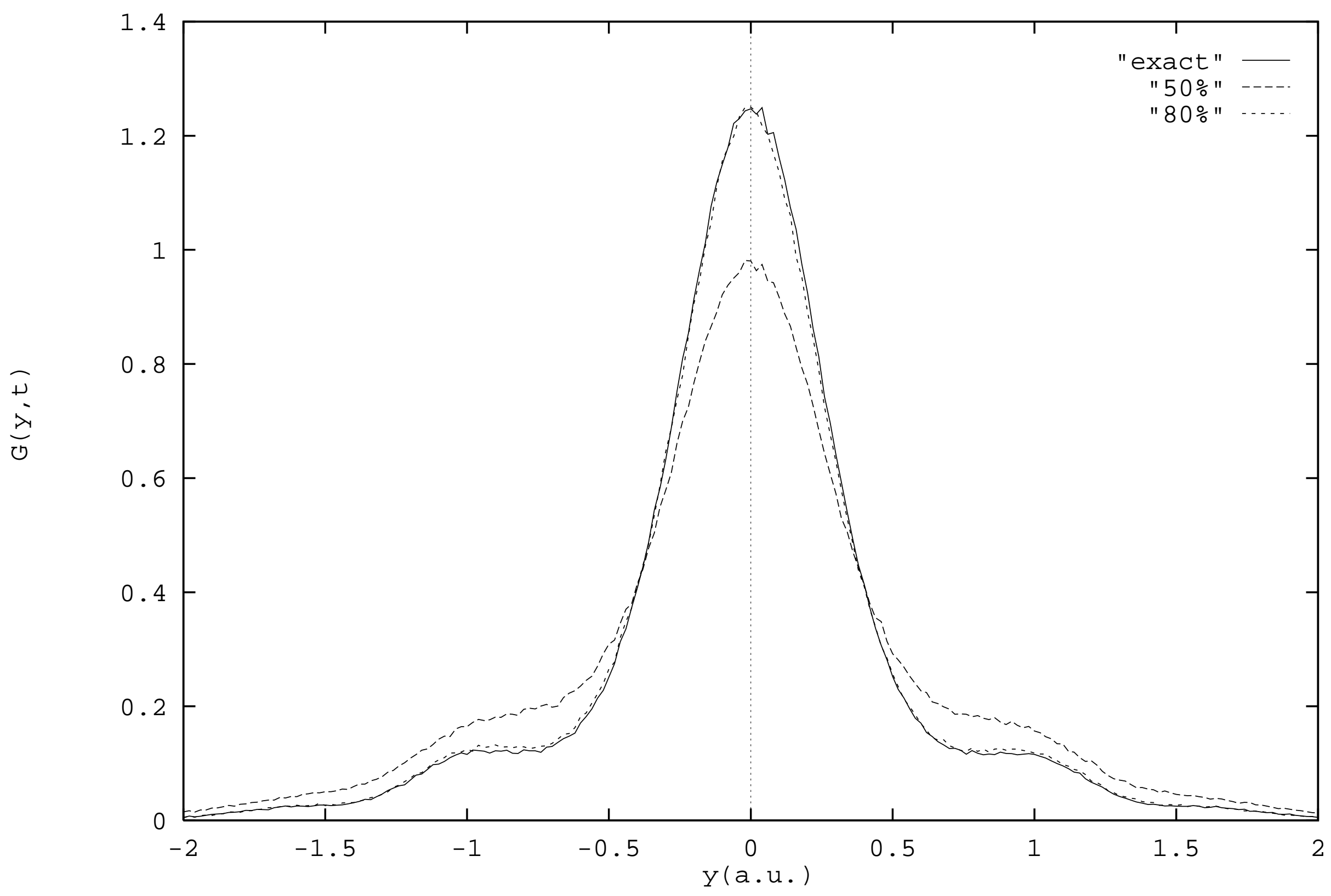

